Service social

\title{
Indicateurs. Une expérience à travers trente ans de réforme de la santé au Québec
}

\section{Hung Nguyen}

Volume 47, numéro 1-2, 1998

Évaluation - Colloque 1999

URI : https://id.erudit.org/iderudit/706783ar

DOI : https://doi.org/10.7202/706783ar

Aller au sommaire du numéro

\section{Éditeur(s)}

École de service social de l'Université Laval

ISSN

1708-1734 (numérique)

Découvrir la revue

Citer cet article

Nguyen, H. (1998). Indicateurs. Une expérience à travers trente ans de réforme de la santé au Québec. Service social, 47(1-2), 91-110.

https://doi.org/10.7202/706783ar

\section{Résumé de l'article}

Se fondant sur une expérience personnelle de trente ans de réforme de santé au Québec, l'auteur retrace l'évolution parallèle du système de santé et des besoins d'indicateurs de gestion. Les besoins d'indicateurs, engendrant les besoins d'information, résultent principalement de deux dynamiques : celle du système administratif et celle du système de services. La difficulté de s'entendre sur les indicateurs clés de gestion reflète-t-elle, à tous les niveaux, un profond malaise dans la gestion du système de santé? Plusieurs expériences novatrices étant en cours, nous décrivons les types d'indicateurs qui peuvent être générés, sans oublier la spécificité des milieux ruraux. Le projet de capitation expérimenté dans la MRC rurale du Haut-Saint-Laurent requiert ainsi un ensemble d'indicateurs qui permet de gérer le système dans une perspective de santé globale. 


\section{Indicateurs \\ Une expérience à travers trente ans de réforme de la santé au Québec ${ }^{1}$}

Hung NGUYEN

Se fondant sur une expérience personnelle de trente ans de réforme de santé au Québec, l'auteur retrace l'évolution parallèle du système de santé et des besoins d'indicateurs de gestion. Les besoins d'indicateurs, engendrant les besoins d'information, résultent principalement de deux dynamiques : celle du système administratif et celle du système de services. La difficulté de s'entendre sur les indicateurs clés de gestion reflète-t-elle, à tous les niveaux, un profond malaise dans la gestion du système de santé? Plusieurs expériences novatrices étant en cours, nous décrivons les types d'indicateurs qui peuvent être générés, sans oublier la spécificité des milieux ruraux. Le projet de capitation expérimenté dans la MRC rurale du Haut-Saint-Laurent requiert ainsi un ensemble d'indicateurs qui permet de gérer le système dans une perspective de santé globale.

From a personal experience of 30 years of health care reforms, the author traces the parallel evolution of the health care system and its management indicators. Within health care, indicators have been developing according to the needs of two distinct but related interests: the administrative system and the service system. Meanwhile population health indicators are developing at a slightly different level. So far, an accepted, integrated set of indicators is lacking. Does this reflect a fundamental problem with the health care system? In Québec, many innovative experiments are undenway; we describe some of the new types of indicators which might result from them. Our capitation project in the rural MRC of Haut-Saint-Laurent requires an integrated set of indicators which unites the financial, service and health information necessary to manage the system in a perspective of global health care.

1. L'auteur remercie Roger Sinclair, de la Régie régionale de la santé et des senvices sociaux de la Montérégie, et Nelson Potvin du MSSS pour leurs suggestions pertinentes, au moment de l'écriture de ce document. Cependant, l'auteur est le seul responsable de son contenu. 


\section{LES INDICATEURS ET LE SYSTÈME REPRÉSENTÉ}

Les indicateurs, ya-t-il sujet plus passionnant? Le terme “ indicateur " me ramène à des événements qui se bousculent dans mes pensées, au fur et à mesure que reviennent mes préoccupations professionnelles relativement au système de santé, qui vont de la mise en œuvre de la réforme Castonguay à l'implantation de la réforme Rochon, aux compressions et, maintenant, à l'ajustement du système. Mon remueméninges rappelle une série de mots, de concepts et de représentations auxquels sont associés les indicateurs. Voici ces éléments, une fois réorganisés :

- ministère de la Santé et des Services sociaux (MSSS), Conseil régional de la santé et des services sociaux (CRSSS), Régie régionale de la santé et des services sociaux (RRSSS), associations, corporations;

- réseau, système, regroupement d'établissements, établissement, organisation, département, services, programmes;

- gestion, gestion intégrée, planification, programmation, mise en œuvre, intervention, prévision, allocation de ressources, suivi de gestion, “ monitoring ", évaluation, qualité, performance;

- santé, social, santé publique, santé communautaire;

- population, maladie, épisode de soins, besoins, demandes, offres, services, ressources, budgets;

- « input », structure, processus, " output », environnement, objectifs, résultats, impacts.

Si chacun de nous refait l'exercice, je suis certain qu'il trouvera d'autres éléments qui lui sont personnels. Nous constatons aussi que ces éléments représentent les systèmes de référence (organisations, structures, concepts, fonctions, théories, approches, etc.) pour lesquels nous utilisons ou définissons des indicateurs. Le choix d'un système de référence reflète le point de vue d'un individu ou d'un groupe; il précède le choix des indicateurs. Autrement dit, les indicateurs ont un sens relatif à leur système de référence et nous ne pouvons pas les interpréter en dehors de ce système.

Notons que : « le système est défini [comme une] vue de l'esprit, une représentation finalisée (subjective) de la réalité. Un système est toujours 
le résultat des choix de l'observateur (théorie, point de vue, etc.) face à la réalité : ceci s'exprime sous la forme d'un découpage de la réalité en système (ce qui est dedans), en environnement (ce qui est dehors) et dans l'expression des objectifs reconnus pour ce système. La structure d'un système est représentée par un modèle (ou un ensemble de modèles de natures diverses)... " (Pascot et collab., 1987).

Les indicateurs qui nous intéressent sont relatifs au système de santé, qui comporte une multitude de systèmes de référence en rapport avec la santé, les services, le réseau, les interventions, l'évaluation et la performance, etc. (Kaplan et Norton, 1996; Sicotte, Champagne et Contandriopoulos, 1999; Rummler et Brache, 1990; Modèle de l'AHQ2; Institut canadien d'information sur la santé, 1999). Cependant, en général, nous distinguons le système administratif du système des services.

Dès la mise en œuvre de la réforme Castonguay, le système administratifest composé de trois paliers de gestion : MSSS, CRSSS, établissements et autres producteurs de services. Quant au système de services, il est constitué essentiellement des établissements, des organismes communautaires, des cabinets des médecins et des autres professions de santé.

Le système administratif. Durant cette période de trente ans suivant la réforme Castonguay, les CRSSS (conseils régionaux de la santé et des services sociaux) deviennent des RRSSS (régies régionales de la santé et des services sociaux). De ce fait, on leur accorde plus de pouvoir quant à la planification, la programmation, l'allocation de ressources et l'évaluation.

Le système de services. Comme tout le monde le sait, ce système passe successivement par des périodes de développement, puis de compressions sévères. Maintenant, un ajustement vers la hausse se prépare. Les acteurs institutionnels ne changent pas beaucoup, mais leur mission se modifie, en particulier celle des CLSC (Centre local des services communautaires) et des hôpitaux.

2. Système informatisé d'analyse de la performance des établissements (SIAP). Ce modèle établit trois niveaux de performance : macro, qui concerne l'établissement dans sa globalité, méso, qui s'intéresse au programme clientèle et micro, qui porte sur les centres d'activités. 
Durant cette période de trente ans de réforme de santé, les besoins d'indicateurs, devenus des besoins d'information, sont principalement les résultats de deux dynamiques : celle du système administratif et celle du système de services. Ces dynamiques s'entrecroisent et sont difficiles à isoler. Mais nous savons que les indicateurs utilisés dans ces deux systèmes diffèrent pour une grande part (Nguyen, 1999) et qu'ils sont en partie dictés par les paliers de gestion supérieurs (dans l'étude portant sur les besoins d'information dans le cadre du tableau de bord de clientèle de CLSC, $50 \%$ des indicateurs requis proviennent des paliers administratifs supérieurs).

Dans l'évolution parallèle du système de services et du système administratif, les indicateurs apparaissaient, disparaissaient et réapparaissaient en fonction des préoccupations des acteurs de l'heure. L'instabilité des indicateurs, d'une part, et la difficulté de s'entendre sur les indicateurs clés de gestion pour le réseau de services, d'autre part, sont-elles le reflet d'un profond malaise dans la gestion du système de santé et ce, à tous les niveaux? Je tente, à travers mon histoire personnelle, de relater une partie de l'évolution de ces deux systèmes, administratifs et de services, ainsi que des indicateurs qui s'y rattachent. C'est un point de vue tout à fait personnel et intuitif.

\section{INDICATEURS ET SYSTÈME DE SANTÉ AU QUÉBEC : TRENTE ANS D'ÉVOLUTION}

\section{Mise en œuvre des recommandations de la commission Castonguay (1971-1976 )}

Formé en économétrie, j'ai eu mon premier contact avec le réseau de la santé en 1971. J'ai présidé un groupe de recherche financé par les deux paliers de gouvernement, composé d'une vingtaine de chercheurs et d'experts. Notre but était de modéliser le système de santé (MEDIC : Modèle d'évaluation des interactions complexes de la santé) (Nguyen, 1976) et de simuler des allocations de ressources. Nous avons travaillé simultanément sur une quinzaine de projets qui s'articulaient autour d'un « macro » modèle ayant comme composantes l'offre, la demande de services et le mécanisme d'allocation de ressources, en fonction de l'écart entre l'offre et la demande. Je reproduis ici le modèle de référence, car les idées de base sont encore valables à différents niveaux de l'organisation du système actuel. 


\section{Modèle de référence}

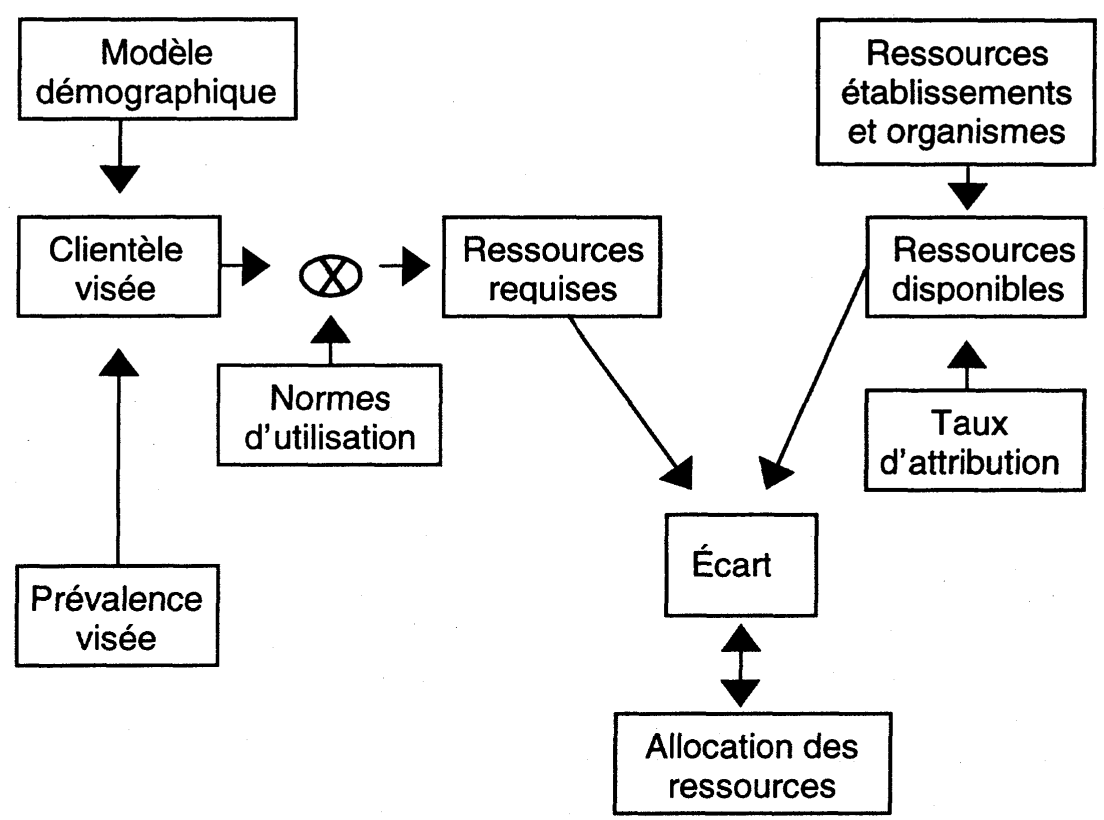

Ce schéma de référence a engendré des travaux importants pour quantifier les composantes du système de représentation et leur évolution. Nous avons travaillé dans cette perspective durant les cinq années subséquentes. Nous avons recensé des indicateurs (le Conseil des affaires sociales et de la famille [1978] a aussi mené une étude importante à ce sujet et certaines questions soulevées à l'époque restent très pertinentes actuellement) et les avons mis en relation pour décrire quantitativement le modèle de référence et prévoir leur dynamique dans le temps. Quelques types d'indicateurs utilisés sont ici mentionnés en bref (voir page suivante). 


\section{Prévision de la demande}

\begin{tabular}{|c|c|c|c|}
\hline $\begin{array}{c}\text { Évolution de la } \\
\text { population }\end{array}$ & $\begin{array}{c}\text { Évolution de la } \\
\text { maladie }\end{array}$ & $\begin{array}{l}\text { Norme d'utilisation } \\
\text { des ressources }\end{array}$ & $\begin{array}{l}\text { Demande de } \\
\text { ressources }\end{array}$ \\
\hline $\begin{array}{l}\text { Indicateurs } \\
\text { démographiques, } \\
\text { par: } \\
\text { - âge, sexe, taux } \\
\text { de mortalité, } \\
\text { taux de natalité, } \\
\text { taux d'immigra- } \\
\text { tion, taux de } \\
\text { mobilité, taux de } \\
\text { rétention et taux } \\
\text { de croissance } \\
\text { de la population }\end{array}$ & $\begin{array}{l}\text { Indicateurs de } \\
\text { maladie selon le } \\
\text { code CIMA } \\
\text { - hospitalisation, } \\
\text { morbidité, } \\
\text { prévalence }\end{array}$ & $\begin{array}{l}\text { Indicateurs moyens } \\
\text { d'utilisation des } \\
\text { ressources : } \\
\text { - lits d'hôpital, } \\
\text { médecins, } \\
\text { infirmières, etc. } \\
\text { Indicateurs de } \\
\text { coûts moyens : } \\
\text { - lits d'hôpital, } \\
\text { médecins, } \\
\text { infirmières, etc. }\end{array}$ & $\begin{array}{l}\text { (Multiplication } \\
\text { des } 3 \text { éléments } \\
\text { précédents) } \\
\text { Indicateurs des } \\
\text { ressources } \\
\text { financières } \\
\text { requises, par: } \\
\text { - catégorie de } \\
\text { ressources, } \\
\text { type de } \\
\text { maladie et } \\
\text { tranche de } \\
\text { population }\end{array}$ \\
\hline
\end{tabular}

\section{Prévision de l'offre}

\begin{tabular}{|c|c|c|}
\hline Ressources actuelles & Taux d'attribution & Ressources disponibles \\
\hline \multirow{4}{*}{$\begin{array}{l}\text { Indicateurs des } \\
\text { ressources actuelles } \\
\text { par: } \\
\text { établissement et type } \\
\text { de spécialité }\end{array}$} & $\begin{array}{l}\text { Indicateurs d'utilisation } \\
\text { actuelle ou visée des }\end{array}$ & $\begin{array}{l}\text { (Multiplication des } 2 \\
\text { éléments précédents) }\end{array}$ \\
\hline & $\begin{array}{l}\text { ressources. Ex. : } \\
\text { - utilisation actuelle des }\end{array}$ & $\begin{array}{l}\text { Indicateurs des ressour- } \\
\text { ces disponibles }\end{array}$ \\
\hline & $\begin{array}{l}\text { lits : } 70 \% \text {, } \\
\text { utilisation visée : } 80 \%\end{array}$ & $\begin{array}{l}\text { coûts par catégorie } \\
\text { de ressources }\end{array}$ \\
\hline & $\begin{array}{l}\text { Indicateurs des coûts } \\
\text { moyens pour une } \\
\text { ressource }\end{array}$ & \\
\hline
\end{tabular}

\section{Allocation de ressources}

\begin{tabular}{lll}
\hline Demandes non satisfaites & Priorité & Allocation des ressources \\
\hline $\begin{array}{l}\text { Indicateurs des demandes } \\
\text { non satisfaites: }\end{array}$ & $\begin{array}{l}\text { Indicateurs de priorité } \\
\text { donnée à chaque type de } \\
\text { demandes non satisfaites }\end{array}$ & $\begin{array}{l}\text { Selon différentes } \\
\text { approches }\end{array}$ \\
$\begin{array}{l}\text { écart entre l'offre et la } \\
\text { demande }\end{array}$ & & \\
\hline
\end{tabular}

3. CIMA : Classification internationale des maladies et des affections. 


\section{Leçon retenue de cette expérience}

$\checkmark$ Les indicateurs ont pour but d'aider à comprendre nos propres systèmes de référence. La question est de savoir si notre système de référence recevrait l'appui des acteurs œuvrant dans le même domaine.

$\checkmark$ La signification des indicateurs est limitée, d'une part, par leur classification (système déjà très complexe : CIMA - Actes médicaux $\mathrm{DRG}^{4}$ - Interventions) et, d'autre part, par les interrelations complexes utilisées pour décrire le comportement des organisations.

Les indicateurs élaborés, de type macro, pouvaient influencer l'évolution permettant d'orienter des politiques, à moyen terme (3 à 5 ans), mais ils ont peu d'impact sur le système opérationnel.

\section{La création des CRSSS}

Plans régionaux, rationalisation des ressources

et équité interrégionale (1976-1989),

début de l'enquête Santé Québec

Jusqu'en 1976, le MSSS gérait directement son réseau d'établissements. II faut comprendre que les indicateurs étaient développés seIon ces deux pôles : Central et Unités de production. J'ai travaillé pour un CRSSS jusqu'à 1989. Cette période se caractérisait par la création et la consolidation de l'identité régionale. C'est alors que, dans les modèles de référence, d'autres dimensions sont apparues : le niveau régional, le niveau géographique, le réseau régional et l'équité inter et intrarégionale.

L'articulation entre les paliers central et régional se faisait, d'une part, par l'intermédiaire des politiques (commission Rochon, 1987, 1988; MSSS, 1989) et, d'autre part, par l'allocation de ressources (le mécanisme était régionalisé de façon progressive) (Bordeleau, 1996; Nguyen, 1996). Il faut noter aussi que, de façon remarquable, la préparation et les recommandations de la commission Rochon ont enrichi les réflexions sur les modèles de référence, les indicateurs et les processus de gestion et de production de senvices. C'est tout à fait normal car, là où il y a de la recherche, il y a de nouvelles données et de nouveaux indicateurs.

4. DRG : Diagnostics Related Group. 
En parlant de la dynamique de gestion par résultats impliquant tous les paliers de gestion, Yvon Brunelle et Alain Saucier (1999) concluent : "L'utilisation des indicateurs ne devrait plus servir seulement à porter un jugement sur la façon dont on dispense les services en bout de piste, mais également sur la façon de planifier et de gérer le réseau sociosanitaire, et ce, à tous les niveaux. Les problèmes de qualité ne se posent pas seulement aux derniers dispensateurs dans la chaîne de soins. "

C'était aussi le coup d'envoi d'un grand mouvement de développement de systèmes d'information pour la gestion efficace du réseau, à tous les échelons. Dès 1989, à coups de millions de dollars, le comité MSSS-CRSSS a lancé des projets de développement de systèmes d'information (Sauvageau, 1996) dans tous les secteurs, mais particulièrement dans le secteur des senvices sociaux. Dès lors sont apparues les préoccupations concernant les processus, le cheminement du client, les indicateurs de gestion et les tableaux de bord (Nguyen, 1999).

Le travail de la commission Rochon portait sur le recensement des indicateurs dans le milieu de la santé et des services sociaux et visait à dégager et à quantifier les informations nécessaires (Pascot et collab., 1987), permettant de constater la complexité des indicateurs employés, selon les nombreux utilisateurs. Les auteurs ont recensé environ 300 indicateurs clés qui étaient utilisés dans le système et qui pourraient être documentés systématiquement.

Enfin, l'enquête de Santé Québec qui débutait en 1987 a créé un tout autre domaine d'indicateurs et de schèmes différents de référence. Les résultats de l'enquête, rendus publics en 1989, ont influencé les travaux portant sur la santé de la population, en particulier au niveau régional (Pampalon et collab., 1990). L'enquête a été reprise en 19921993 et en 1998.

Durant la période «CRSSS », qui a duré 13 ans, le développement des indicateurs était influencé par quelques éléments, tels que :

- la dimension régionale et géographique;

- la coordination des établissements à l'intérieur d'une région;

- l'équité des ressources entre les établissements, les programmes et les régions (MSSS, 1987); 
- le développement des services des CLSC dans les domaines suivants : maintien en milieu de vie naturel, santé mentale, services pour personnes en perte d'autonomie et jeunesse;

- la mise en œuvre des politiques du gouvernement et du MSSS, à la suite des recommandations de la commission Rochon;

- le mouvement de développement des systèmes d'information (qui se poursuit toujours) et des tableaux de bord.

Cependant, durant cette période, on note une absence : les CRSSS ne participaient jamais à la gestion des services médicaux sauf en ce qui a trait à la planification des effectifs, ce qui est contraire à son rôle dans la gestion des ressources institutionnelles. On a observé une dichotomie dans la gestion des services médicaux et des services institutionnels. Seuls les services institutionnels sont régionalisés. Cette " absence " persiste encore. Depuis 30 ans, on « observe " ce problème. Je ne connais pas de plans, ni de programmes émanant soit du MSSS ou soit des régies régionales visant la mise en œuvre d'une gestion intégrée des services de première ligne ou une intégration clinique des services de première ligne. Cette situation explique-t-elle qu'actuellement il existe peu de coordination entre les services médicaux, sociaux et institutionnels?

D'autres lacunes reposent, d'une part, sur l'absence d'instruments efficaces pour la mise en commun des ressources des établissements pour la même clientèle et, d'autre, part, sur la coordination des soins pour un même patient, surtout celui en perte d'autonomie ou vivant un problème de santé mentale (l'implantation mitigée des plans de services individualisés, $P S I$, persiste jusqu'à maintenant). Et, enfin, y a-t-il lieu de revoir la responsabilité des établissements relativement à la santé d'une population définie géographiquement?

Nous allons voir deux nouveaux termes, l'un issu du rapport de la commission Rochon, "Programme clientèle " (Maranda, 1996), et l'autre de la loi, "les plans régionaux de services ", qui, durant les dix années subséquentes, ont engendré d'immenses efforts concertés dans l'ensemble du réseau de la santé en vue de la création d'outils de gestion, d'indicateurs et de systèmes d'information. Nous constaterons que ces travaux ont permis de progresser dans la création des outils de gestion, sans toutefois régler trois problèmes fondamentaux du système de santé, à savoir : 
- Responsabilité des producteurs à l'égard de la santé de la population (élargir le rôle de production de services vers une responsabilité de santé qui pourra atténuer la demande).

- Coordination des services aux patients (perte d'autonomie, santé mentale) requérant des services interétablissements (actuellement, ce rôle de coordination est laissé aux intervenants des établissements, mais cette démarche de concertation se heurte aux différentes barrières administratives et professionnelles - voir Nguyen, 1997, sur la réingénierie des processus collectifs dans Châteauguay et autres régions).

- Coordination entre les services médicaux, sociaux et institutionnels, surtout en première ligne.

\section{Régies régionales}

Programmes clientèle, PROS, plan de coordination horizontale, objectifs de santé, performance, compressions, évaluation, intégration de la santé publique (1990-1997)

En 1990, les CRSSS sont devenus les RRSSS que nous connaissons. Au cours des sept années subséquentes, les RRSSS ont implanté plusieurs approches de gestion visant la coordination horizontale et verticale des services.

Ainsi, le mouvement d'élaboration et d'implantation des PROS (Plan régional d'organisation de services) était lancé dans toutes les régions avec le PROS Santé mentale.

Par la suite, on a tenté d'étendre les PROS à tous les programmes clientèle : “ un ensemble d'activités intégrées destinées à une clientèle particulière ou à une population, visant des résultats spécifiques pour lesquels sont consenties des ressources humaines, matérielles et financières ${ }^{5} »$. Une nomenclature de programmes a été créée pour baliser les clientèles du système de santé (Sauvageau, 1996). Les programmes clientèle ont aussi servi d'instruments de gestion de résultats pour le MSSS et les RRSSS et, par la suite, ils ont servi à

5. Comité MSSS-Régies sur la gestion par programmes, 1994. 
allouer des ressources aux régions ou encore à répartir les compressions. Marc-André Maranda (1996) a bien décrit cette époque dans son article "Planification centrale et régionale en santé et services sociaux : Confusion autour du dispositif ».

Les PROS (Berthiaume, 1996) et les programmes clientèle ont fourni l'occasion formidable de développer des indicateurs dans tous les domaines :

- Indicateurs de besoins (le concept de besoins est abstrait et réfère à une perturbation de la santé et du bien-être qui empêche les individus de bien remplir les fonctions et les rôles (Pampalon et collab., 1995, p. 6). Le terme " indicateurs de besoins " réfère à l'une des trois approches pour mesurer les besoins, à savoir : l'approche d'indicateurs, d'enquête et recherche de consensus (Pampalon, Nguyen et Francœur, 1990, p. 14).

- Indicateurs de services : ce sont les équivalents des besoins qui s'expriment par les services (voir schéma adapté de Donabedian dans Pampalon, Nguyen et Francœur, 1990).

- Indicateurs de ressources : ce sont les équivalents des besoins en ressources.

- Indicateurs de gestion (Nguyen, 1996) : l'expression renvoie aux mesures qui permettent d'observer la réalisation d'un objectif d'amélioration d'une organisation.

- Indicateurs de suivi et d'évaluation (Mercier et Corin, 1993); ils correspondent, en général, aux mesures qui permettent de faire le suivi et l'évaluation d'une intervention, d'une organisation ou d'un système.

Certaines régions ont même déterminé les ressources nécessaires pour une intervention ou pour des services en santé mentale (RSSSMontégérie, 1992; Tremblay, 1993).

Le MSSS et les RRSSS ont tenté une approche de gestion par programmes clientèle tant dans l'organisation de services que dans l'allocation des ressources. Le MSSS a créé aussi une banque de données financières assorties d'indicateurs de besoins et de performance pour allouer les ressources aux régions, et ce, par programmes (voir l'opération Défi-Qualité-Performance, MSSS, 1993). 
Le PROS Santé mentale a aussi été évalué par le Groupe de recherche interdisciplinaire en santé - Université de Montréal (GRIS) (Denis et collab., 1996). La conclusion décrivait la place des outils de gestion (dont les indicateurs) dans la gestion des programmes clientèle.

Les responsables de l'opération PROS et de la planification en général sont à la recherche d'outils formalisés de gestion pour assumer leurs nouvelles responsabilités en matière d'allocation de ressources.

Les interrogations portent principalement sur les caractéristiques des systèmes d'information à mettre en place, sur les indicateurs à développer pour baliser l'allocation des ressources et la représentation des besoins et sur les systèmes d'évaluation à implanter pour piloter le réseau sociosanitaire avec une certaine rationalité $[. .$.

Par ailleurs, la réforme semble se dérouler aussià l'extérieur de la planification, au sens le plus classique du terme. Cet outil (PROS) est insuffisant pour assurer linfluence de nouvelles logiques et de nouveaux acteurs dans l'organisation des services en santé mentale.

II faut noter que les PROS et les programmes clientèle sont des outils de gestion du MSSS et des RRSSS. Toutefois, ils engendrent une tâche importante, soit celle de la collecte de données du système opérationnel. Imaginons un établissement, tel un CLSC, qui devrait intégrer toutes les politiques du MSSS, les plans et les programmations provenant de la RRSSS, et, en plus, produire des indicateurs de toutes sortes, en particulier les ententes MSSS-RRSSS ${ }^{6}$. Apparaît donc le paradoxe entre le système administratif et le système de services. De plus, le MSSS et les RRSSS produisent et implantent les politiques et les plans, obligeant ainsi les établissements à consacrer du temps et des ressources aux aspects administratifs du système de santé. Alors, où est l'équilibre?

Le MSSS a lancé la Politique de la santé et du bien-être (1992) à peu près en même temps que les programmes clientèle. La politique se dotait d'une vingtaine d'objectifs de santé assujettis à des résultats à atteindre et à des indicateurs qui permettent de suivre la réalisation des objectifs de santé. Les indicateurs de santé prennent leur essor.

6. Protocoles d'entente entre MSSS et Régies, 1999. Ces protocoles spécifient les objectifs de résultats à atteindre et les indicateurs de suivi à utiliser. Les objectifs sont différents selon les régions. 
Mais il y a toujours eu une conciliation difficile entre le système de santé et le système de services. Les deux systèmes fonctionnent avec des schèmes de référence différents, avec des systèmes d'information et d'indicateurs différents; donc, l'intégration dans une même approche de gestion semble peu probable. Patricia Caris (1996) a discuté de cet aspect délicat qu'est l'organisation du système de santé et des services de santé, à savoir : La distinction et la conciliation au même ministère et même réseau : l'action sur les déterminants de la santé et du bien-être (système de santé) et la responsabilité de l'accès aux services sociosanitaires de qualité (système de soins).

En 1993, le MSSS a commencé ses compressions dans le réseau avec l'opération " Défi-Qualité-Performance ». La compression a été répartie entre les régions selon une approche de l'équité interrégionale se basant sur les " per capita par programme ». Pour aider les régions à réaliser les compressions, le MSSS a aussi déterminé et fourni aux régions le niveau de gains réalisables pour chaque établissement, si ce dernier fonctionnait comme la moyenne d'un groupe d'établissements comparables ou encore s'il devait atteindre un seuil visé de performance. La compression était faite par récupération des gains réalisables. Jusqu'en 1997, cette opération a provoqué un mouvement important de rationalisation dans le système, et ce, à tous les niveaux. Nous réfléchissons alors sur la performance, la qualité et d'autres manières de faire (réingénierie de processus [Nguyen et Rho, 1996; Nguyen et collab., 1997a; Nguyen et collab. 1997b; Lafrenière et collab. 1998], système de gestion intégrée, réseau de services intégrés, fusion, coordination interétablissements, etc.).

Les PROS, qui devaient être un outil de développement de ressources et d'affirmation régionale, deviennent alors " l'instrument de compressions ", exactement le contraire des objectifs visés au moment de leur élaboration.

\section{Maintenant et l'avenir (1998-...)}

\section{Financement par capitation et approche territoriale?}

Comme nous le savons, les compressions et le départ massif du personnel et d'employés cadres créent une situation de déséquilibre dans le système de santé au Québec. Les acteurs se redéfinissent donc par rapport à leur propre mission et à celle des autres acteurs, afin de retrouver l'équilibre. II y aura sûrement des travaux en ce sens et 
s'ajouteront d'autres indicateurs et d'autres systèmes de référence. Nous entendons parler déjà " du nouveau rôle de l'hôpital, pôle de coordination de services à la population ${ }^{7}$ ", des essais par rapport à d'autres modes de fonctionnement (mégacliniques, centre ambulatoire, réseau de services intégrés) et de financement (projets de capitation SIPA et MRC de Haut-Saint-Laurent), du projet d'organisation locale de services intégrés de financement par capitation (projet de la MRC du Haut-Saint-Laurent pour l'ensemble de la population et projet SIPA pour personnes âgées) et de la responsabilité des services envers une population (projet de capitation MRC du Haut-Saint-Laurent).

De plus, nous parlons déjà de réinvestissements dans le système de santé et, lorsqu'il est question de réinvestissements, tout de suite nous pensons aux coûts, à la performance et aux résultats. C'est l'ère de la " sanction de la performance " ou encore " des ententes de gestion visant les résultats entre le MSSS et les RRSSS » :

Ces ententes comportent des objectifs et des cibles spécifiques, convenus mutuellement, en matière d'accès aux services, de gestion de ressources, d'implantation de nouveaux programmes et d'élimination des déficits courants... Ces cibles sont assorties à des indicateurs de mesure (Mercier et Camirand, 1999).

Le débat actuel sur les services de santé et l'innovation technologique est centré sur les régions urbaines; on discute moins des milieux ruraux où des problématiques existent en permanence, à savoir : le manque permanent de ressources professionnelles (faible probabilité de trouver des solutions permanentes pour les années à venir) et le retard par rapport au milieu rural dans l'adaptation technologique (actuellement, dans plusieurs établissements les opérations cliniques de base ne sont pas « informatisées »). Dans ces conditions, ces milieux devront créer leur propre modèle, plus flexible à l'égard des pratiques professionnelles et organisationnelles, d'une part, et s'assurer de moyens pour soutenir son fonctionnement, d'autre part. Est-il souhaitable de fournir aux milieux ruraux les pouvoirs d'organisation et de financement pour créer des outils de gestion adaptés pour eux? C'est ici que l'approche territoriale et l'organisation des services locaux sont pertinentes.

Si l'organisation des services de santé est un élément explicatif de la santé de la population, nous avons encore beaucoup à faire pour inciter

7. Voir Le Devoir, 6 octobre 1999. 
les organismes locaux à se coordonner dans la prise en charge des patients et à se donner une responsabilité à l'égard de la santé de la population locale. Ce dernier élément est très déficient dans le système de santé actuel. C'est dans cette perspective que nous expérimentons un projet de capitation dans la MRC du Haut-Saint-Laurent. Par ce projet, nous tentons de développer les indicateurs adaptés au contexte d'évaluation du fonctionnement d'un système de santé, au niveau local, en lien avec le processus clinique, la qualité des soins et la satisfaction de la population et des autres acteurs du système (Contandriopoulos et collab., 1999).

\section{Discussion}

Au cours des trente dernières années, malgré les essais visant à modifier le système de référence, à replacer les acteurs et à redéfinir les règles du jeu, les problèmes fondamentaux persistent, à savoir :

- La jonction entre le système de santé et le système de services. Cette question appelle la définition des responsabilités des producteurs à l'égard de la santé de la population. Actuellement, nous tentons d'opérer ce rapprochement au niveau régional par l'intermédiaire de la politique de santé. Est- ce que le niveau local est préférable pour opérer ce rapprochement et, si oui, quels seront les outils (administratifs, financiers, cliniques et informationnels) nécessaires?

- La coordination entre les établissements. Un grand nombre de patients ont besoin de services de plusieurs établissements, par exemple les personnes en perte d'autonomie et celles qui ont des problèmes de santé mentale. La coordination des services interétablissements augmente l'efficacité et la qualité des services pour ces patients et permet de réduire les coûts. Le financement par établissement ne favorise pas cette coopération souhaitée. Peut-on envisager d'autres modes de financement, par exemple sur une base de capitation ou encore les projets interétablissements visant une population définie?

- Le rapprochement des pratiques professionnelles. Ce rapprochement est important pour l'ensemble du système de santé. II est vital pour les régions rurales où le problème de recrutement est 
permanent et où des pratiques plus flexibles permettant de s'adapter à la situation sont à inventer.

- La disponibilité des données financières et cliniques d'un patient. La performance des établissements pourra dépasser le concept d'épisode de soins par établissement pour introduire les concepts de patients interétablissements ou par programme. En même temps, il faudra accroître l'information pertinente pour soutenir cette approche (voir projet de capitation de la MRC du Haut-SaintLaurent, banque de données intégrées).

- La coordination centrale, régionale et locale. La performance du système est systémique et implique une définition des objectifs clairs à chaque niveau afin d'apprécier leur efficacité. Au cours des trente dernières années, beaucoup d'efforts ont été déployés afin d'articuler le niveau régional, mais qu'en est-il actuellement? Il est question d'un modèle hiérarchique entre MSSS, régies régionales et établissements ou bien d'un modèle à deux niveaux où les opérations de la régie régionale sont commandées par le MSSS ou, encore, à trois niveaux où chaque instance maintient ses relations avec les deux autres, selon les mandats et les missions spécifiques.

- Finalement, ce que la population attend est simple. C'est l'accessibilité à des services de qualité, donc : un accueil courtois, des soins dans un délai acceptable et une relation satisfaisante avec les cliniciens. Quant à l'aspect clinique de l'intervention, la plupart d'entre nous faisons " confiance " aux cliniciens.

Des indicateurs en ce sens devront-ils être développés? Et, enfin, les expériences au Québec, en particulier celles qui ont trait aux intégrations de services ou en capitation, peuvent-elles apporter des éclaircissements aux questions soulevées?

\section{CONCLUSION}

Le système de santé a de multiples facettes et modèles de référence. L'histoire des indicateurs illustre à quel point il est difficile de définir la place de chacun (individu, groupe, organisation, palier administratif et système) dans l'organisation du système de santé et sa participation à l'atteinte de l'objectif collectif, à savoir : un système de services intégrés de qualité. 
Bien au-delà des systèmes complexes de référence associés au système de santé, les soins sont d'abord constitués par les interventions cliniques, dans les processus recourant à différentes disciplines pour répondre aux besoins des patients. Les résultats obtenus par le patient sont les conséquences directes de la qualité de ces processus cliniques intra et interétablissements; qualité qui découle d'abord du niveau de compétence des ressources humaines. On retrouve ici le cadre d'analyse systémique : structure, processus, résultats. Le défi est que les indicateurs que nous utilisons reflètent une partie seulement d'une vision systémique de l'organisation des services de santé.

Autour de cet aspect fondamental s'articulent différents volets administratifs à des fins de planification (santé, santé publique, planification sociale, communautaire, stratégique, tactique, opérationnelle), de programmation, d'allocation de ressources, de suivi, de « monitoring ", d'évaluation et de recherche, ainsi que de " monitoring " administratif. Ces autres aspects sont importants, certes, mais ils sont tous liés à des systèmes de référence qui sont, par définition, restrictifs, personnels (selon le point de vue) et temporels (à changer selon les préoccupations et les cibles). II faudra doser le développement de ces indicateurs et mesurer les efforts requis par les établissements pour les produire.

Hung NGUYEN

Cadre-conseil

Planification et développement

Direction de la santé publique, de la planification et de l'évaluation

Régie régionale de la santé et des services sociaux de la Montérégie 


\section{Références bibliographiques}

BERTHIAUME, Nicole (1996). "Les plans régionaux d'organisation de services : Des outils pour la gestion des programmes ", dans Mireille Tremblay, Hung Nguyen et Jean Turgeon, La planification régionale des services sociaux et de santé, Québec, Les Publications du Québec.

BORDELEAU, LuC (1996). “ Orientations et priorités régionales », dans Mireille Tremblay, Hung Nguyen et Jean Turgeon, La planification régionale des services sociaux et de santé, Québec, Les Publications du Québec.

BRUNeLLE, Yvon et Alain SAUCIER (1999). Les indicateurs et le système de soins, Québec, ministère de la Santé et des Services sociaux, Direction générale de la planification et de l'évaluation.

CARIS, Patricia (1996). "Planification et transformation : comment concilier les deux bouts de la lorgnette? ", dans La planification régionale des services sociaux et de santé, Québec, Les Publications du Québec.

Commission d'enquête sur les services de santé et les services sociaux (Commission Rochon, 1987-1988). Rapport de la commission d'enquête sur les services de santé et les services sociaux, Québec, Les Publications du Québec.

CONSEIL DES AFFAIRES SOCIALES ET DE LA FAMILLE (1978). La question des indicateurs.

CONTANDRIOPOULOS, André-Pierre, Jean-Louis Denis, Claude Sicotte et collab. (1999). Projet de capitation de la MRC du Haut-Saint-Laurent : Protocole d'évaluation, Département d'administration de la santé, Université de Montréal.

DENIS, Jean-Louis et collab. (1996). "La planification et le changement : Analyse de l'expérience des PROS dans le système de santé au Québec ", dans La planification régionale des services sociaux et de santé, Québec, Les Publications du Québec.

GASCON, Richard, Denis TURGEON et Hung NGUYEN (1999). Conférence sur " Développer et implanter des mesures de performance dans le domaine de santé " à Montréal, 21 et 22 septembre 1999, session « La marche à suivre et les meilleurs outils pour assurer le développement d'indicateurs de mesures de performance valables ", Institut canadien, Toronto, Ontario.

KAPLAN, Robert S. et David P. NORTON (1996). “ Using the Balanced Scorecard as a Strategic Management System ", Harvard Business Review, janvierfévrier, Boston, USA.

LAFRENIÈRE, Mario et collab. (1998). Réingénierie des services de maintien en milieu de vie naturel dans la sous-région de Saint-Hyacinthe, Longueuil, Régie régionale de la santé et des services sociaux, Montérégie. 
MARANDA, Marc-André (1996). «Planification centrale et régionale en santé et services sociaux : Confusion autour du dispositif ", dans La planification régionale des services sociaux et de santé, Québec, Les Publications du Québec.

MERCIER, C. et E. CORIN (1993). La pertinence des indicateurs sociosanitaires pour l'élaboration et le suivi des plans régionaux d'organisation de services, Colloque sur les enjeux politiques et méthodologiques de la planification régionale des services sociaux et de la santé, 61 e congrès de l'ACFAS, Rimouski, Département de psychiatrie de l'Université de Montréal et Centre de recherche de l'Hôpital Douglas.

MERCIER, Jean et François CAMIRAND (1999). L'amélioration de la performance hospitalière : vision et rôle du MSSS, ministère de la Santé et des Services sociaux, conférence prononcée lors du colloque organisé par l'Institut canadien les 21 et 22 septembre à Montréal sur « Développer et implanter les mesures de performance dans le domaine de la santé ".

Ministère de LA SANTÉ ET DES SERVICES SOCIAUX (1989). Pour améliorer la santé et le bien-être au Québec : Orientations, Québec, Gouvernement du Québec, 147 p.

MINISTÈre DE LA SANTÉ ET DES SERVICES SocIaUX (1989). Politique de santé mentale, Québec, Gouvernement du Québec.

MINISTÈRE DE LA SANTÉ ET DES SERVICES SOCIAUX (1989). Une réforme axée sur le citoyen, Québec, Gouvernement du Québec, $91 \mathrm{p}$.

MINISTÈRE DE LA SANTÉ ET DES SERVICES SOCIAUX (1987). Modifications suggérées aux critères d'allocation de ressources consenties par le MSSS au réseau des affaires sociales, Québec, MSSS, Direction générale de la planification et de l'évaluation.

NGUYEN, Hung (1999). Tableau de bord de la clientèle de CLSC : Son rôle dans le développement des indicateurs de performance du système québécois de santé, conférence prononcée les 21 et 22 septembre, Institut canadien.

NGUYEN, Hung (1996). Expérience pilote : Gestion par programme clientèle en santé mentale, Longueuil, Régie régionale de la santé et des services sociaux.

NGUYEN, Hung (1976). Projet MEDICS : Modèle d'évaluation des interractions complexes de la santé, Québec, ministère de la Santé et des Services sociaux, Direction de la planification.

NGUYEN, Hung et collab. (1997). Réingénierie de services en milieu de vie naturel dans la sous-région de Saint-Jean, Longueuil, Régie régionale de la santé et des services sociaux, Montérégie. 
NGUYEN, Hung et collab. (1997). Réingénierie des services de maintien en milieu de vie naturel dans la sous-région de Châteauguay, Longueuil, Régie régionale de la santé et des services sociaux, Montérégie.

NGUYEN, Hung et Guy RHO (1996). Réingénierie des services d'alimentation dans la sous-région de Longueuil, Longueuil, Régie régionale de la santé et des services sociaux, Montérégie.

PAMPALON, Robert et collab. (1995). Des indicateurs de besoins pour l'allocation interrégionale des ressources, Groupe technique sur les indicateurs de besoins, Québec, ministère de la Santé et des Services sociaux.

PAMPALON, Robert et collab. (1990). La santé à la carte, Québec, Les Publications du Québec.

Pampalon, Robert, Hung NguYen et Danièle Francoeur (1990). Cadre de référence et stratégie d'élaboration d'indicateurs de besoins pour l'allocation régionale des ressources, Québec, ministère de la Santé et des Services sociaux (Méthodologie et Instrumentation).

Pascot, Daniel, Claude BANVILle, Hung NGuYEN, Robert MANTHA et Dzenan RIDJANOVIC (1987). Bilan critique et cadre conceptuel des systèmes d'information dans le domaine de la santé et des services sociaux, Rapport de recherche $n^{\circ} 26$, Québec, Commission Rochon.

RUMmLER, Geary A. et Alan P. BRACHE (1990). Improving Performance, how to manage the white space on the organization chart, San Francisco, CA, Jossey-Bass Publishers.

SAUVAGEAU, André-Guy. " La planification régionale et les systèmes d'information ", dans La planification régionale des services sociaux et de santé, Québec, Les Publications du Québec.

SicotTE, Claude, François ChAMPAGnE et André-Pierre CONTANDRIOPOULOS (1999). "La performance organisationnelle des organismes publics de santé ", Ruptures, revue transdisciplinaire en santé, GRIS, Université de Montréal, Montréal.

TREMBLAY, Mireille, Hung NGUYEN et Jean TURGEON (1996). La planification régionale des services sociaux et de santé, Québec, Les Publications du Québec.

TREMBLAY, Mireille (1993). Un modèle de répartition des ressources par programme : À la recherche de l'équité entre la mesure et la concertation, Colloque sur les enjeux politiques et méthodologiques de la planification régionale des services sociaux et de santé, $61^{\mathrm{e}}$ congrès de l'ACFAS à Rimouski. 